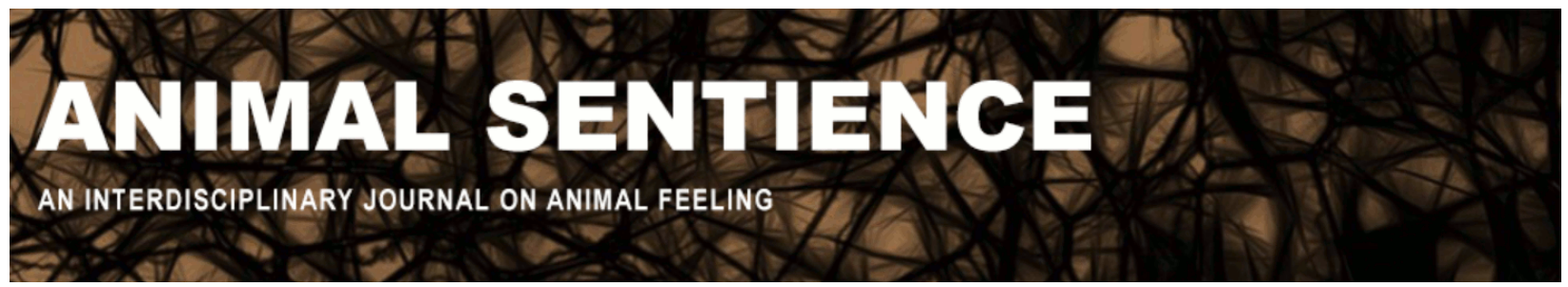

Bräuer, Juliane; Silva, Karine; and Schweinberger, Stefan R. (2017) Communicating canine and human emotions. Animal Sentience 14(7)

DOI: 10.51291/2377-7478.1244

Date of submission: 2017-11-06

Date of acceptance: 2017-11-08

(c) 


\title{
Communicating canine and human emotions
}

Commentary on Kujala on Canine Emotions

\author{
Juliane Bräuer ${ }^{1,3}$, Karine Silva², and Stefan R. Schweinberger ${ }^{1}$ \\ ${ }^{1}$ Department of General Psychology and Cognitive Neuroscience, FSU, Jena, Germany \\ ${ }^{2}$ Departamento de Ciências do Comportamento, Instituto de Ciências Biomédicas \\ Abel Salazar, Universidade do Porto, Portugal \\ ${ }^{3}$ Max-Planck-Institut for the Science of Human History, Jena, Germany
}

\begin{abstract}
Kujala (2017) reviews a topic of major relevance for the understanding of the special dog-human relationship: canine emotions (as seen through human social cognition). This commentary draws attention to the communication of emotions within such a particular social context. It highlights challenges that need to be tackled to further advance research on emotional communication, and it calls for new avenues of research. Efforts to disentangle emotional processes from cognitive functioning might be necessary to better comprehend how they contribute, alone and/or in combination, to the communication of emotions. Also, new research methods need to be developed to account for the rich sensory repertoire of dogs, likely involved in emotional communication.
\end{abstract}

Juliane Bräuer is a researcher at the University of Jena and the Max-Planck-Institute for the Science of Human History. Her research area is Comparative Psychology and she is especially interested in investigating the cognitive skills that different species - particularly dogs - have evolved to survive in their ecological niche. Her research topics include communication, cooperation, perception and individual differences.

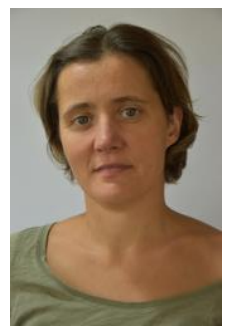

doglab.shh.mpg.de/dog-cognition.php

Karine Silva holds a Ph.D. in Biology and is currently working as a post-doctoral researcher in the field of Anthrozoology at the Institute of Biomedical Sciences Abel Salazar of the University of Porto. She is especially interested in determining whether positive, emotional, interactions with dogs may promote health improvements, notably for social dysfunctions. sigarra.up.pt/up/en/web base.gera pagina?p pagina=icbas

Stefan R. Schweinberger is Professor of General Psychology at the University of Jena. His research is on the cognitive neuroscience of person perception, recognition by face and voice, human interaction processes, and the brain mechanisms, individual differences and disorders associated with these abilities. www.allgpsy.uni-jena.de/stefan-r-schweinberger/

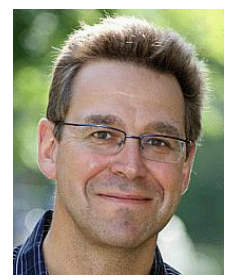


In the last 20 years, there has been growing scientific interest in the domestic dog and its role in its special niche: the human environment (Kaminski \& Marshall-Pescini 2014, Miklosi 2007). Most studies have focused on the cognitive skills dogs evolved in the context of that environment. Investigating canine emotions is important in understanding this special doghuman relationship. Kujala (2017) has reviewed an up-to-date topic, which - as she points out - is difficult to investigate because humans readily impute emotion to others. She is very careful in interpreting the results and also in adding relevant comparative findings from cognitive neuroscience. In our comment, we focus on the communication of emotion in both species, proposing new research directions and approaches.

1. How humans perceive emotions: Individual differences. In her second section, Kujala describes data from studies focusing on how humans classify dogs' emotions. Human participants usually have to match photos, video clips, or sound recordings to the appropriate situations (i.e., Bloom \& Friedman 2013, Pongracz et al. 2005; Mirkó et al. 2005). Usually the results are interpreted to indicate that participants were able to classify the dog's emotion - although it should be noted that we can only infer that dogs in these situations were actually angry, fearful or sad. Kujala emphasizes this point in her introduction, but it tends to get lost in the subsequent discussion of empirical studies. One disadvantage of these studies is that the stimuli are often snapshots of a given situation, or sound recordings or, in a few studies, video clips. These stimuli have limited ecological validity - after all, dogs and humans do not perceive emotion by vision or audition alone; they use all available modalities, including olfaction.

When humans classify human emotions, it is unlikely that facial stimuli, such as the classical Ekman faces (Ekman \& Friesen 1971) are perceived by everyone in the same way (Matsumoto 1990). In a recent study, Gendron et al. (2014) asked participants from the US and from the Himba ethnic group (a nomadic tribe in Northern Namibia) to sort images of facial expressions by emotion type. Himba participants did not show the same sorting pattern, thus suggesting that how emotion is perceived from facial expressions is not culturally universal.

Also within cultures, there are individual differences in humans' ability to process human emotions (Hamann \& Canli 2004). For instance, there is evidence that facial imitation is unconsciously taking place when we view other people's emotions (Dimberg et al. 2000), and that the degree of imitation may be inversely related to autistic tendencies (Lewis \& Dunn 2017). Thus, the understanding of another human's emotional nonverbal signs depends, at least to some degree, on empathy (Dziobek et al. 2008). These considerations may inform future research on emotional communication in the particular context of human-dog interaction.

2. How dogs perceive emotions: The multimodal approach. As Kujala (2014) points out, a number of recent studies have investigated how dogs perceive human emotions. For example, dogs can discriminate emotional expressions of human faces on a touch screen (Müller et al. 2015). Moreover, dogs orient toward a person more often when the person is pretending to cry than just talking or humming (Custance \& Mayer 2012). D'Aniello et al. (2017) report that dogs are sensitive to human body odours produced under two different emotional conditions. Bräuer et al. (2013) found that dogs help humans when these showed 
signs of needing support desperately. In another study, however, dogs failed to help when their owners feigned a heart attack (Macpherson \& Roberts 2006).

These studies investigated only a single aspect or modality of the detection of human emotion by dogs and the perceived emotions were not "real," in that they were either only feigned by humans or only depicted in pictures. Future studies should use more holistic approaches in which humans really feel the emotions. (This can be achieved, for example, by mood induction techniques.) With the opportunity to use the visual, the auditory and olfactory modalities, dogs may prove to be much more accurate in detecting human emotions. It has been suggested that when dogs' ancestors - wolves - must decide which individuals in a herd of prey to hunt, it would be adaptive for them to perceive which are sick or especially fearful (see also Gadbois \& Reeve 2014). Dogs have been shown to be able to detect various diseases in humans such as cancer and diabetes (Lim et al. 1992, Lippi \& Cervellin 2012); and therapy dogs seem to react differently toward patients depending on their disorders (Prothmann et al. 2004). This sensitivity might also enable them to detect human emotions.

There are probably also individual differences in dogs' ability to perceive human emotion (Arden \& Adams 2016; Miklósi et al. 2014). We believe that dogs are also a good candidate species to test whether the perceptions and the expression of emotions are closely interlinked via common internal representations in the embodiment of emotion (Niedenthal 2007).

3. Smell and emotion. A very interesting thought of Kujala's is that based on their rich sense of smell - and their reliance on it in exploring the environment and recognizing individuals - dogs may have unique emotional states. As most current research is limited to the visual or auditory modality, it is not yet clear how olfaction, emotion and cognition are linked. As it is difficult for humans to imagine what it is like to have such an excellent nose, it is a challenge to test it.

Human studies may nevertheless point the way. We know that the brain regions that process smell are phylogenetically old and linked to limbic areas of the brain as well as memory areas. Steinberg et al. (2012) used whole-head magnetoencephalography (MEG) in humans to measure brain responses to more than 100 faces with neutral expression, each associated with either an aversive or a neutral odour via classical conditioning. Powerful and remarkably olfactory conditioning to these faces was reflected in enhanced early (50-80 ms and 130-190 ms after face onset) neuronal responses. These findings reflect a capacity for (implicit) emotional learning in the absence of awareness. As dogs rely so much on olfaction, such mechanisms may be even more efficient in them.

4. The cognitive component. An important question is how emotion and cognition are linked. Kujala states that there are probably cognitive limits to empathy in dogs. Similarly, Bräuer (2015) has pointed out that dogs are highly motivated to behave pro-socially toward people but often lack the cognitive capacity to understand how to help the human partner.

Cognitive limits appear to occur mainly on the receiver side. A very recent study suggests that, on the sender side, dogs show a surprising flexibility. Kaminski et al. (2017) tested whether dog facial expressions are subject to audience effects - or whether they only depend on arousing stimuli such as food. A human demonstrator was either paying attention to the tested dog or turned away, and she either presented food or not. Dogs produced significantly more facial movements when the human was attentive than when she was not. 
By contrast, food as a non-social but arousing stimulus did not affect the dogs' facial behaviour. The authors concluded that facial expressions are not just inflexible and involuntary displays of emotional states; they are potentially active attempts to communicate with humans (Kaminski et al. 2017).

When humans were attentive, dogs showed one facial movement in particular, the so-called inner eyebrow raise that changes the visual appearance of the eyes and makes them look bigger. The authors argue that the eyebrow raise either resembles a facial movement that in humans indicates sadness (making humans feel more empathic toward dogs who produce this movement more) or it is a feature of paedomorphism (as humans have a preference for child-like characteristics). Waller et al. (2013) found that dogs from a shelter that produced this facial movement were rehomed often more quickly.

These promising approaches lead to further questions about the communicative role of emotional expressions in dogs' faces, how they are perceived by humans, and how this has influenced the process of domestication. Overall, we expect that future progress in understanding canine (and human) emotional communication will benefit from a consideration of systematic individual differences, from further exploring links between the perception and expression of emotion, and from novel and ecologically valid tests of the rich multisensory nature of the representational capacities of dogs' brains.

\section{References}

Arden, R. \& Adams, M. J. (2016) A general intelligence factor in dogs. Intelligence 55: 79-85.

Bloom, T. \& Friedman, H. (2013) Classifying dogs' (Canis familiaris) facial expressions from photographs. Behavioural Processes 96: 1-10.

Bräuer, J. (2015) I do not understand but I care: The prosocial dog. Interaction Studies 16(3): 341360.

Bräuer, J., Schönefeld, K. \& Call, J. (2013) When do dogs help humans? Applied Animal Behaviour Science 148(1-2): 138-149.

Custance, D. \& Mayer, J. (2012) Empathic-like responding by domestic dogs (Canis familiaris) to distress in humans: An exploratory study. Animal Cognition 15(5): 851-859.

D’Aniello, B., Semin, G. R., Alterisio, A., Aria, M. \& Scandurra, A. (2017) Interspecies transmission of emotional information via chemosignals: From humans to dogs (Canis lupus familiaris). Animal Cognition.

Dimberg, U., Thunberg, M. \& Elmehed, K. (2000) Unconscious facial reactions to emotional facial expressions. Psychological Science. Psychological Science 11: 86-89.

Dziobek, I., Rogers, K., Fleck, S., Bahnemann, M., Heekeren, H. R., Wolf, O. T. \& Convit, A. (2008) Dissociation of cognitive and emotional empathy in adults with asperger syndrome using the Multifaceted Empathy Test (MET). Journal of Autism and Developmental Disorders 38(3): 464-473.

Ekman, P. \& Friesen, W. V. (1971) Constants across cultures in the face and emotion. Journal of Personality and Social Psychology 17: 124-129.

Gadbois, S. \& Reeve, C. (2014) Canine olfaction: Scent, sign, and situation. In: Domestic Dog Cognition and Behavior, eds. A. Horowitz, Springer.

Gendron, M., Roberson, D., van der Vyver, J. M. \& Barrett, L. F. (2014) Perceptions of emotion from facial expressions are not culturally universal: Evidence from a remote culture. Emotion 14(2): $251-262$. 
Hamann, S. \& Canli, T. (2004) Individual differences in emotion processing. Current Opinion in Neurobiology 14: 233-238.

Kaminski, J., Hynds, J., Morris, P. \& Waller, B. M. (2017) Human attention affects facial expressions in domestic dogs. Scientific Reports 7(1): 12914.

Kaminski, J. \& Marshall-Pescini, S. (2014) The Social Dog: behaviour and cognition, Elsevier publishers.

Kujala, M. V. (2017) Canine emotions as seen through human social cognition. Animal Sentience 14(1).

Lewis, M. B. \& Dunn, E. (2017) Instructions to mimic improve facial emotion recognition in people with sub-clinical autism traits. The Quarterly Journal of Experimental Psychology 70(11): 2357-2370.

Lim, K., Fisher, M. \& Burns-Cox, C. J. (1992) Type 1 diabetics and their pets. Diabetic Medicine 9: S3-S4.

Lippi, G. \& Cervellin, G. (2012) Canine olfactory detection of cancer versus laboratory testing: Myth or opportunity? Clinical Chemistry and Laboratory Medicine 50(3): 435-439.

Macpherson, K. \& Roberts, W. A. (2006) Do dogs (Canis familiaris) seek help in an emergency? Journal of Comparative Psychology 120(2): 113-119.

Matsumoto, D. (1990) Cultural similarities and differences in display rules. Motivation and Emotion 14(3): 195-214.

Miklosi, A. (2007) Dog behaviour, evolution, and cognition, Oxford University Press

Miklósi, A., Turcsán, B. \& Kubinyi, E. (2014) The personality of dogs. In: The social dog: Behaviour and cognition, eds. J. Kaminski \& S. Marshall-Pescini.

Mirkó, E., Dóka, A. \& Miklósi, Á. (2013) Association between subjective rating and behaviour coding and the role of experience in making video assessments on the personality of the domestic dog. Applied Animal Behaviour Science 149(1): 45-54.

Müller, Corsin A., Schmitt, K., Barber, Anjuli L. A. \& Huber, L. (2015) Dogs can discriminate emotional expressions of human faces. Current Biology 25(5): 601-605.

Niedenthal, P. M. (2007) Embodying emotion. Science 316(5827): 1002.

Pongracz, P., Molnar, C., Miklosi, A. \& Csanyi, V. (2005) Human listeners are able to classify dog (Canis familiaris) barks recorded in different situations. Journal of Comparative Psychology 119(2): 136-144.

Prothmann, A., Schaumberg, A. \& Ettrich, C. (2004) The diagnostic value of pet facilitated therapy with dogs. Society of Companion Animal Studies Journal 16(1): 3-6.

Steinberg, C., Dobel, C., Schupp, H., Kissler, J., Elling, L., Pantev, C. \& Junghöfer, M. (2012) Rapid and highly resolving: Affective evaluation of olfactory conditioned faces. Journal of Cognitive Neuroscience 24(1): 17-27.

Waller, B. M., Peirce, K., Caeiro, C. C., Scheider, L., Burrows, A. M., McCune, S. \& Kaminski, J. (2013). Paedomorphic facial expressions give dogs a selective advantage. PLOS ONE, 8(12), e82686. 


\section{ANIMAL CONSCIOUSNESS}

On November 17-18, 2017, the NYU Center for Mind, Brain and Consciousness, the NYU Center for Bioethics, and NYU Animal Studies will host a conference on Animal Consciousness.

This conference will bring together philosophers and scientists to discuss questions such as: Are invertebrates conscious? Do fish feel pain? Are nonhuman mammals self-conscious? How did consciousness evolve? How does research on animal consciousness affect the ethical treatment of animals? What is the impact of issues about animal consciousness on theories of consciousness and vice versa? What are the best methods for assessing consciousness in nonhuman animals?

\section{Speakers and panelists include:}

Colin Allen (University of Pittsburgh, Department of History \& Philosophy of Science), Andrew Barron (Macquarie, Cognitive Neuroethology), Victoria Braithwaite (Penn State, Biology), Peter Carruthers (Maryland, Philosophy), Marian Dawkins (Oxford, Zoology), Dan Dennett (Tufts, Philosophy), David Edelman (San Diego, Neuroscience),

Todd Feinberg (Mt. Sinai, Neurology), Peter Godfey-Smith (Sydney, Philosophy), Lori Gruen (Wesleyan, Philosophy), Brian Hare (Duke, Evolutionary Anthropology), Stevan Harnad (Montreal, Cognitive Science), Eva Jablonka (Tel Aviv, Cohn Institute), Björn Merker (Neuroscience), Diana Reiss (Hunter,

Psychology), Peter Singer (Princeton, Philosophy), Michael Tye (Texas, Philosophy)

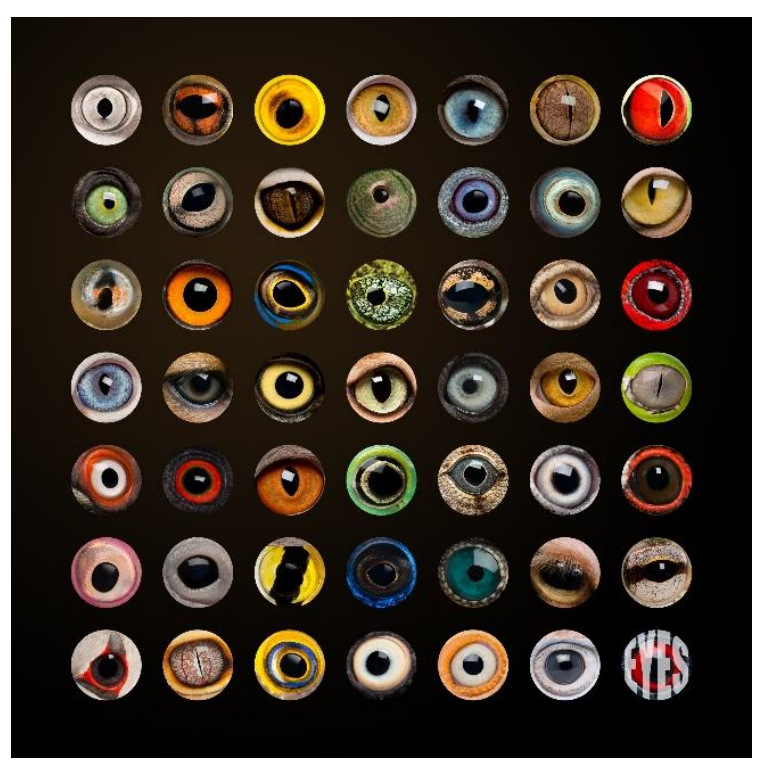

Organizers: Ned Block, David Chalmers, Dale Jamieson, S. Matthew Liao.

The conference will run from 9am on Friday November 17 to $6 \mathrm{pm}$ on Saturday November 18 at the NYU Cantor Film Center (36 E 8th St).

Friday sessions will include "Invertebrates and the evolution of consciousness", "Do fish feel pain?", and "Animal consciousness and ethics".

Saturday sessions will include "Animal self-consciousness", "Animal consciousness and theories of consciousness", and a panel discussion.

A detailed schedule will be circulated closer to the conference date.

Registration is free but required.

\section{Register here.}

\section{See also the conference website}

